\title{
Indicadores de produção de soja no Rio Grande do Sul comparados ao zoneamento agrícola
}

\author{
Ricardo Wanke de Melo(1), Denise Cybis Fontana ${ }^{(2)}$ e Moacir Antonio Berlato(2)
}

\begin{abstract}
(1)Universidade de São Paulo, Escola Superior de Agricultura "Luiz de Queroz", Dep. de Ciências Exatas, Av. Páduas Dias, 11, Caixa Postal 9, CEP 13418-900 Piracicaba, SP. E-mail: wanke@esalq.usp.br (2)Universidade Federal do Rio Grande do Sul, Fac. de Agronomia, Dep. de Plantas Forrageiras e Agrometeorologia, Av. Bento Gonçalves, 7712, Caixa Postal 776, CEP 91540-000 Porto Alegre, RS. E-mail: dfontana@vortex.ufrgs.br, moacir.berlato@ufrgs.br
\end{abstract}

Resumo - O Estado do Rio Grande do Sul é responsável por aproximadamente $20 \%$ da produção nacional de soja, o que torna necessário um conhecimento mais amplo de indicadores da produção desta cultura neste Estado. O objetivo deste trabalho foi analisar, em comparação ao zoneamento agrícola, alguns indicadores de produção da soja nos municípios que compõem a região de produção significativa da cultura da soja no Rio Grande do Sul. A análise de agrupamento foi realizada pelo método de Ward. Os municípios foram agrupados em função do rendimento, da produção e da razão entre a área colhida de soja e a área total do Município. O resultado da análise de agrupamento quanto ao rendimento foi comparado ao zoneamento agroclimático da soja para o Estado. Existe uma estreita relação entre os grupos formados pela análise das três variáveis. O zoneamento agrícola da soja no Estado do Rio Grande do Sul apresenta uma possível inconsistência no agrupamento dos municípios por rendimento. Nos outros agrupamentos, esta inconsistência não é observada.

Termos para indexação: Glycine max, análise de agrupamento, área, produtividade.

\section{Soybean production pointers in Rio Grande do Sul compared to the agricultural zoning}

\begin{abstract}
The State of Rio Grande do Sul is responsible for approximately $20 \%$ of the national soybean production. For this reason better knowledge about pointers of this crop production in this State becomes necessary. The objective of this work was to analyse, comparing to the agricultural zoning, some soybean production pointers in the counties that are part of the signicant production region of this crop in Rio Grande do Sul. The grouping analysis was carried by the Ward's method. Counties were grouped according to the yield, the production and the ratio of the soybean harvested area and the total county area. The yield grouping analysis result was compared to the soybean agroclimatic zoning for the State. There is narrow relation among the groups formed by the three variables analysis. The soybean agroclimatic zoning in the State of Rio Grande do Sul presents a possible inconsistency in the grouping of the counties by yield. In the other groupings, this inconsistency is not observed.
\end{abstract}

Index Terms: Glycine max, grouping analysis, area, yield.

\section{Introdução}

O Rio Grande do Sul é um dos maiores produtores e exportadores de grãos do Brasil, sendo responsável por aproximadamente $20 \%$ da produção nacional de soja. No ano agrícola 2000/2001 foram produzidas $6.935 \mathrm{mil}$ toneladas, com produtividade média de $2.339 \mathrm{~kg} \mathrm{ha}^{-1}$ (IBGE, 2001) e um valor de cerca de 2,3 bilhões de reais (EMATER, 2002).

Entre 1997 e 2000, a cultura da soja apresentou a maior área de cultivo no Rio Grande do Sul, ou seja, em torno de 3 milhões de hectares. Esta cultura participa da economia de pequenos, médios e grandes estabelecimentos rurais, estando presente em $33,14 \%$ destes. Dos estabelecimentos rurais que cultivam soja, $93,94 \%$ possuem áreas com menos de 50 ha, de acordo com o Censo Agropecuário do Estado do Rio Grande do Sul (IBGE, 2001).

Em média, a produção de soja tem sido crescente nos últimos anos em conseqüência da aplicação de tecnologia, uso de materiais genéticos de maior potencial produtivo e da crescente profissionalização dos produtores rurais. No entanto, na maior parte dos anos, a frequiência e a intensidade das chuvas no período do 
desenvolvimento da soja, que ocorre entre novembro e março no Rio Grande do Sul, são insuficientes para que a cultura manifeste seu potencial produtivo (Matzenauer et al., 2002). Assim, o clima é o principal fator responsável pelas oscilações de produtividade e frustrações da cultura da soja no Estado. Estima-se que $93 \%$ das perdas na safra de soja ocorram em razão das estiagens (Berlato \& Fontana, 2003). Este elevado risco faz com que as taxas de sinistralidade na agricultura sejam muito altas. Segundo Cunha \& Assad (2001), nas culturas de verão, esta taxa é, em média, de 16,27\%.

Ávila et al. (1996) analisaram a probabilidade da precipitação pluvial superar a evapotranspiração potencial (P>ETP), no período de cultivo das culturas de primavera-verão no Estado e constataram que, na metade norte, estas probabilidades são maiores do que na metade sul. Mas, em todo o Estado, a baixa probabilidade da precipitação pluvial superar a evapotranspiração potencial, especialmente nos meses de dezembro, janeiro e fevereiro, torna evidente a necessidade de planejamento quanto a épocas de semeadura e quanto à utilização de técnicas de irrigação.

Estes resultados corroboram os de Cunha et al. (2001), que analisaram o risco de ocorrência de deficiência hídrica para a cultura da soja pelo índice ISNA (índice de satisfação das necessidades hídricas), o qual representa o quociente entre ETR, evapotranspiração real, e ETm, evapotranspiração máxima. Os autores concluíram que a disponibilidade hídrica limita a expressão do potencial de produtividade da soja no Rio Grande do Sul. Mesmo no norte do Estado, onde o risco é menor e onde se concentra a produção de soja, este índice assume valores críticos, especialmente a oeste da região.

Assim, são fundamentais à atividade agrícola os trabalhos de zoneamentos agrícolas que visam à redução de riscos de perdas por adversidades climáticas na agricultura, mediante a determinação de regiões e épocas nas quais a semeadura de determinada cultura está sujeita a menores riscos. Entre os trabalhos que tratam da cultura da soja no Rio Grande do Sul, destacam-se os de Mota et al. (1974), Rio Grande do Sul (1994), Cunha \& Hass (1996) e Cunha et al. (2001). A deficiência hídrica é o fator prioritário na definição das zonas mais adequadas ao cultivo da soja no Estado.

O objetivo deste trabalho foi analisar, em comparação ao zoneamento agrícola, alguns indicadores de produção da soja nos municípios que compõem a região de produção significativa desta cultura no Estado do Rio Grande do Sul.

\section{Material e Métodos}

A região de estudo corresponde à área de significativa produção de soja do Estado do Rio Grande do Sul, definida por Berlato \& Fontana (1999), e é responsável, em média, por $90 \%$ do total da produção da cultura da soja no Estado. Localiza-se entre as latitudes $27^{\circ} 4^{\prime} \mathrm{S}$ e

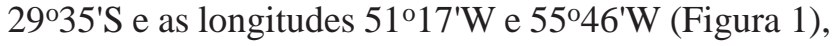
compreendendo 210 municípios de acordo com a divisão do ano de 2000.

Os valores de produtividade média, área colhida e produção de soja dos municípios da região de estudo, referentes ao período de 1981 a 2000, foram obtidos do Instituto Brasileiro de Geografia e Estatística (IBGE, 2001). Em relação a esse período, foram obtidas as informações de área municipal e dados relativos às datas da emancipação do município ou municípios de origem da região de estudo (Federação das Associações de Municípios do Rio Grande do Sul, 2001).

Nas análises de agrupamento foram utilizados os dados somente do período de 1997 a 2000, em virtude da descontinuidade da série de dados, como consequiência do número elevado de emancipações municipais ocorridas na região.

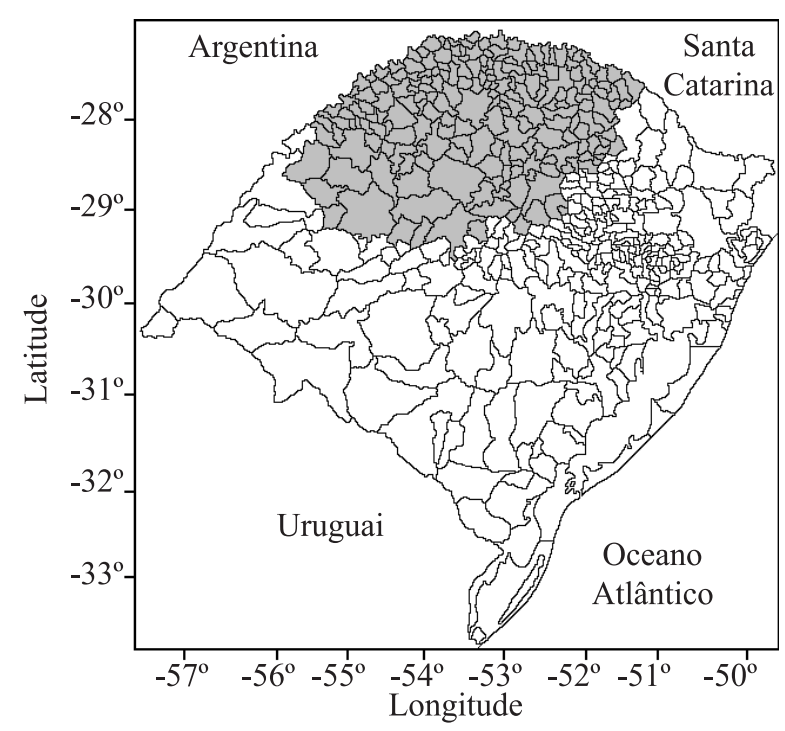

Figura 1. Municípios do Estado do Rio Grande do Sul. Em cinza está representada a região de produção significativa de soja. Fonte: Berlato \& Fontana (1999). 
A análise de agrupamento foi realizada pelo método de Ward, utilizando como medida de dissimilaridade a distância Euclidiana (Wilks, 1995). Os municípios foram agrupados quanto à produtividade, produção e razão entre a área colhida de soja e a área total do município. A análise dos dados e divisão dos grupos foi feita pelo programa SPSS, sendo o resultado dos agrupamentos obtido na forma de dendrogramas com níveis de agrupamentos equivalentes ao número de municípios analisados. Foi realizado um corte nos dendrogramas de maneira que fossem definidos três grandes grupos de cada variável de agrupamento.

Em cada grupo foram calculados os valores médios, máximos e mínimos das variáveis de agrupamento. Os resultados dos agrupamentos foram representados na forma de mapas, para uma melhor visualização da distribuição espacial dos grupos.

Nos agrupamentos gerados em função das produtividades médias, foi confeccionado um gráfico representando a variação temporal da média da variável em cada grupo no período de 1981 a 2000.

O resultado da análise de agrupamento quanto à produtividade média foi comparado ao zoneamento agroclimático da soja (Rio Grande do Sul, 1994), que é baseado no risco de ocorrência de deficiência hídrica e na soma térmica durante o período de crescimento da cultura.

\section{Resultados e Discussão}

Os três grupos de cada variável de agrupamento que foram divididos a partir dos dendrogramas estão relacionados a alta, média e baixa produtividade de soja $\left(\mathrm{kg} \mathrm{ha}^{-1}\right)$, alta, média e baixa produção de soja (toneladas) e alto, médio e baixo porcentual de área cultivada com soja (razão entre a área cultivada com soja e a área total do Município).

\section{Agrupamento de acordo com a produção de soja}

O resultado deste agrupamento evidenciou que os municípios que apresentaram maior produção de soja (grupo $\mathrm{P}_{\mathrm{A}}$ ) encontram-se no centro-sul da região de estudo (Figura 2). Já os municípios que apresentaram menor produção de soja (grupo $\mathrm{P}_{\mathrm{C}}$ ) encontram-se na periferia da região e os municípios que apresentaram média produção de soja (grupo $P_{B}$ ) não apresentaram localização específica, estando distribuídos por toda a região.

Ao ser comparada a divisão dos grupos com o zoneamento agrícola para a cultura da soja no Estado
(Rio Grande do Sul, 1994), verifica-se que os municípios maiores produtores encontram-se, em sua grande maioria, na zona Preferencial-I, e alguns na zona Tolerada-II. Se comparado ao zoneamento proposto por $\mathrm{Cu}$ nha et al. (2001), toda a região de maior produção localiza-se dentro da zona Preferencial, exceto o Município de Santiago.

A maior produção observada nos municípios que compõem o grupo $\mathrm{P}_{\mathrm{A}}$ pode ser conseqüência da maior área cultivada, possivelmente pelo fato de os municípios encontrarem-se, majoritariamente, dentro da zona caracterizada como preferencial pelos zoneamentos agrícolas. Mais a leste, a maior produção pode ser resultado de maior rendimento obtido por unidade de área, como exposto adiante.

As produções, máxima do grupo $\mathrm{P}_{\mathrm{C}}$ e média do grupo $\mathrm{P}_{\mathrm{B}}$, são inferiores à produção mínima do grupo $\mathrm{P}_{\mathrm{A}}$ (Tabela 1). Em média, a produção do grupo $\mathrm{P}_{\mathrm{A}}$ foi de 67.000 toneladas, cerca de onze vezes maior do que a do grupo de menor produção $\left(\mathrm{P}_{\mathrm{C}}\right)$. Cruz Alta foi o $\mathrm{Mu}$ nicípio que apresentou o máximo de produção, atingindo 233.100 toneladas em 1998.

\section{Agrupamento de acordo com o porcentual de área cultivada com soja}

Os municípios que apresentam maior porcentual de área colhida de soja em relação à área total do município (grupo $\mathrm{A}_{\mathrm{A}}$ ) estão localizados na parte cen-

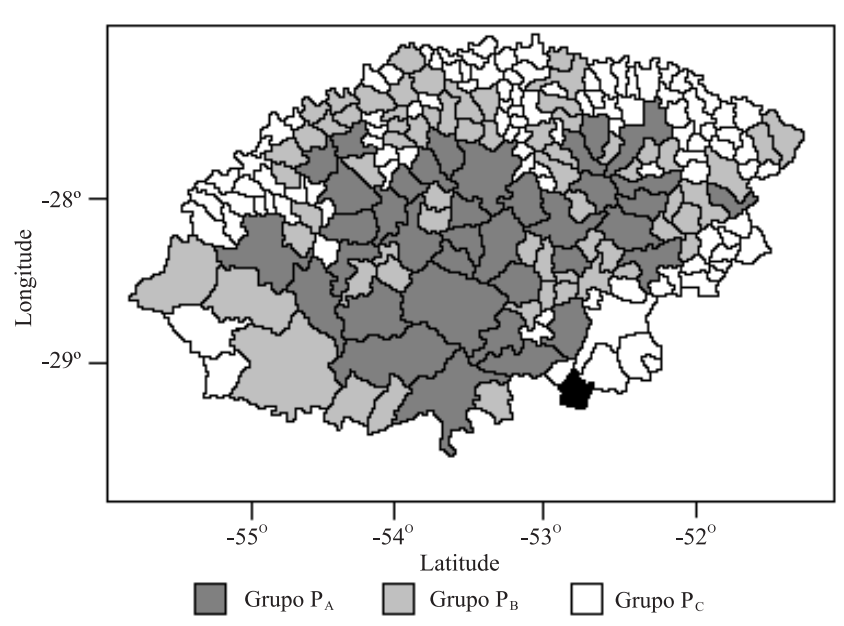

Figura 2. Municípios da região de produção significativa de soja no Rio Grande do Sul agrupados pelo método de Ward, considerando a produção de grãos. O município em preto corresponde a Lagoão. Período: 1997-2000 (Fonte: IBGE, 2001). 
tral da região de estudo e são municípios que possuem áreas médias (Figura 3). Em torno destes, localizam-se os municípios com médio porcentual de área colhida de soja (grupo $A_{B}$ ), e na periferia da região encontram-se os municípios com menor área colhida de soja em termos percentuais (grupo $\mathrm{A}_{\mathrm{C}}$ ).

A boa definição espacial dos grupos formados nesta análise pode ter sido determinada pela tradição dos municípios e dos agricultores no cultivo da soja. Mesmo que as condições para o cultivo não sejam as mais

Tabela 1. Valores médio, máximo e mínimo da produção da soja na região de produção significativa desta cultura no Rio Grande do Sul, no período 1997-2000.

\begin{tabular}{|c|c|c|c|}
\hline $\begin{array}{l}\text { Grupo e } \\
\text { estatística }\end{array}$ & $\begin{array}{l}\text { Produção } \\
\text { (toneladas) }\end{array}$ & Município & Ano \\
\hline \multicolumn{4}{|l|}{ Grupo $\mathrm{P}_{\mathrm{A}}$} \\
\hline Média & 66.740 & & \\
\hline Máximo & 233.100 & Cruz Alta & 1998 \\
\hline Mínimo & 27.000 & Entre-Ijuís & 2000 \\
\hline \multicolumn{4}{|l|}{ Grupo $\mathrm{P}_{\mathrm{B}}$} \\
\hline Média & 22.348 & & \\
\hline Máximo & 54.900 & Bossoroca & 1998 \\
\hline Mínimo & 8.100 & Rio dos Índios & 1999 \\
\hline \multicolumn{4}{|l|}{ Grupo $\mathrm{P}_{\mathrm{C}}$} \\
\hline Média & 5.778 & & \\
\hline Máximo & 24.300 & Vitória das Missões & 1998 \\
\hline Mínimo & 4 & Mariano Moro & 2000 \\
\hline
\end{tabular}

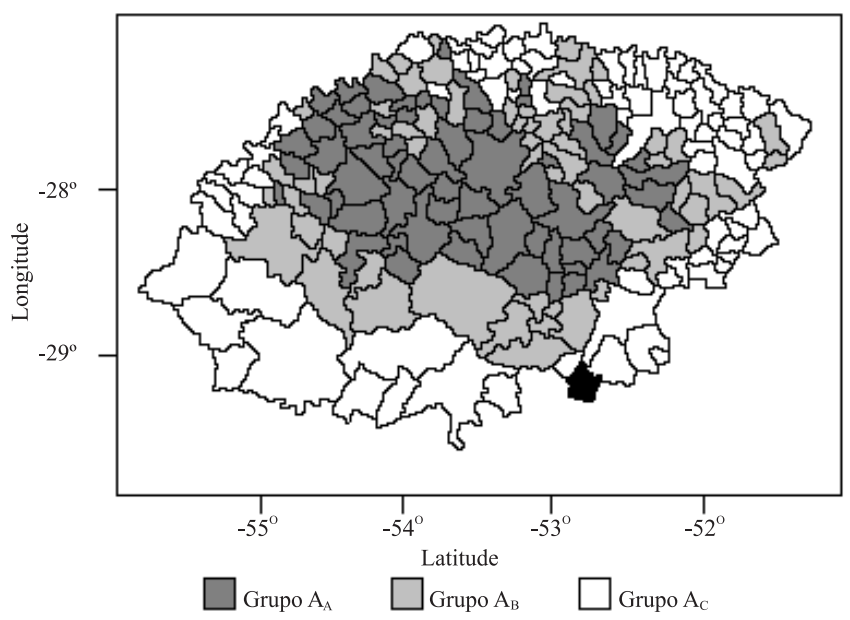

Figura 3. Municípios da região de produção significativa de soja no Rio Grande do Sul agrupados pelo método de Ward, considerando o porcentual de área cultivada com soja. O município em preto corresponde a Lagoão. Período: 1997-2000 (Fonte: IBGE, 2001). adequadas, ou que o município não esteja localizado em área preferencial definida pelo zoneamento agrícola, muitas vezes o produtor prefere não modificar a sua atividade agrícola.

Municípios que apresentam baixa razão $(0,16$ em média) entre a área cultivada com soja e a área total do município possivelmente possuem pequena área agricultável, devido às limitações impostas pela topografia, solos ou outros fatores. O tamanho da propriedade também pode influenciar na definição dos grupos, uma vez que quanto menor a área da propriedade rural, maior a probabilidade de que o produtor diversifique a sua produção. Assim, é possível que o restante da área agricultável do município seja cultivada com outras culturas, principalmente o milho.

No grupo de maior porcentual entre área colhida e área do município, a área cultivada com soja, em média, ocupa mais da metade das áreas dos municípios, demonstrando a importância do cultivo da soja nesta região (Tabela 2).

\section{Agrupamento de acordo com a produtividade mé- dia de soja}

O resultado da análise de agrupamento quanto à produtividade gerou grupos bem definidos e com municípios contíguos (Figura 4). Os grupos $\mathrm{PR}_{\mathrm{A}}, \mathrm{PR}_{\mathrm{B}} \mathrm{e}$ $\mathrm{PR}_{\mathrm{C}}$ corresponderam aos municípios que apresentaram maiores, médias e menores produtividades, respectivamente.

Tabela 2. Valores médio, máximo e mínimo do porcentual de área cultivada com soja e área total do município, na região de produção significativa desta cultura no Rio Grande do Sul, no período 1997-2000.

\begin{tabular}{lccc}
\hline $\begin{array}{l}\text { Grupo e } \\
\text { estatística }\end{array}$ & $\begin{array}{c}\text { Área } \\
\text { relativa }(\%)\end{array}$ & Município & Ano \\
\hline Grupo A & & & \\
Média & 58,06 & Humaitá & 2000 \\
Máximo & 84,64 & Boa Vista das Missões & 1997 \\
Mínimo & 41,68 & & \\
Grupo A & & & 1997 e 1998 \\
Média & 41,87 & Nova Candelária & 1997 \\
Máximo & 59,04 & Lajeado do Bugre \\
Mínimo & 13,89 & & 1997 \\
Grupo A & & & 2000 \\
Média & 16,06 & Palmitinho \\
Máximo & 41,18 & Mariano Moro & \\
Mínimo & 0,04 & \multicolumn{3}{c}{}
\end{tabular}


Os municípios com menores produtividades localizam-se na porção oeste da região de estudo, enquanto as porções norte e centro-sul apresentaram produtividades médias e a porção centro-leste apresentou as maiores produtividades. A definição destas áreas pode ser atribuída a elementos climáticos, solo (material de origem, fertilidade, capacidade de armazenamento de água e outros) ou manejo da cultura (cultivar e outros).

Existe uma variação das produtividades médias na região de estudo, sendo a diferença entre as produtividades médias da região de maiores produtividades e da região de menores produtividades de aproxima-

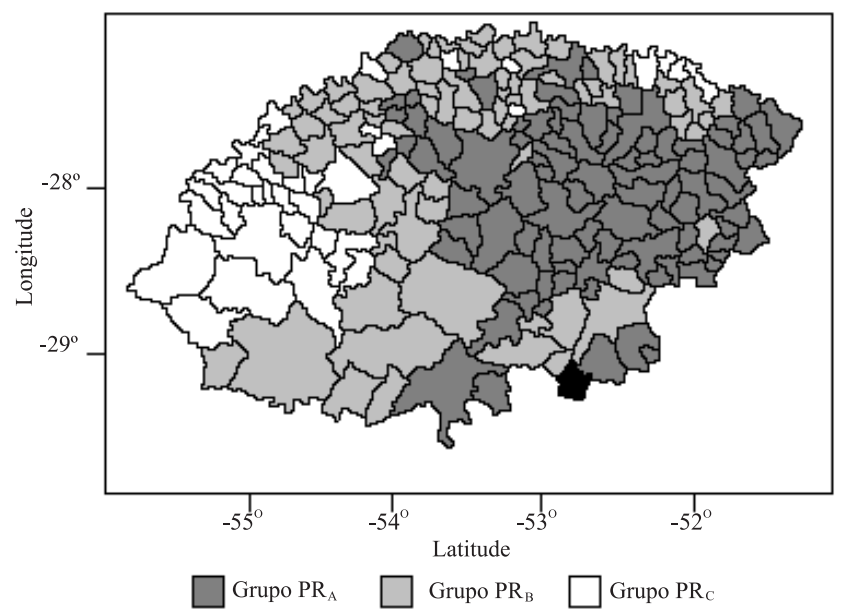

Figura 4. Municípios da região de produção significativa de soja no Rio Grande do Sul agrupados pelo método de Ward, considerando a produtividade. O município em preto corresponde a Lagoão. Período: 1997-2000 (Fonte: IBGE, 2001). damente $800 \mathrm{~kg} \mathrm{ha}^{-1}$ (Tabela 3). Isto é importante nos anos de estiagem, quando esta diferença é maior do que a produtividade máxima obtida em muitas lavouras.

$\mathrm{O}$ grupo $\mathrm{PR}_{\mathrm{B}}$ (médias produtividades) ocupou a maior parte da zona Preferencial-I, onde, segundo o zoneamento agroclimático, não existem restrições ao desenvolvimento da cultura soja (Figura 5). Já o grupo $\mathrm{PR}_{\mathrm{A}}$ (maior produtividade) ocupou a maior parte da zona Tolerada-II, onde a restrição ao desenvolvimento da soja se dá por insuficiência térmica, reduzindo a estação de crescimento da cultura. Esta aparente inconsistência pode evidenciar que a temperatura exerce maior influência na duração do ciclo da cultura do que na produtividade final de grãos. Neste caso, a utilização de variedades precoces adaptadas às condições térmicas da região evita reduções nas produtividades. $\mathrm{O}$ grupo $\mathrm{PR}_{\mathrm{C}}$ (menores produtividades) localizou-se principalmente na zona Tolerada-III, com restrições por deficiência hídrica, principal fator promotor da redução das produtividades de grãos (Cunha \& Bergamaschi, 1992).

Cunha et al. (2001) propuseram um novo zoneamento para a soja no Rio Grande do Sul, com base, principalmente, na disponibilidade hídrica para a soja. As regiões com maiores probabilidades de ocorrência de deficiência hídrica ou menor ISNA (índice de satisfação das necessidades de água) estão localizadas, com maior freqüência, na parte oeste da região de produção significativa de soja no Rio Grande do Sul. Mais a leste, o risco diminui, com valores de ISNA maiores. Portanto, os resultados obtidos mos-

Tabela 3. Valores médio, máximo e mínimo da produtividade da soja na região de produção significativa no Rio Grande do Sul no período 1997-2000.

\begin{tabular}{|c|c|c|c|}
\hline $\begin{array}{l}\text { Grupo e } \\
\text { estatística }\end{array}$ & $\begin{array}{l}\text { Produtividade } \\
\qquad\left(\mathrm{kg} \mathrm{ha}^{-1}\right)\end{array}$ & Município & Ano \\
\hline \multicolumn{4}{|l|}{ Grupo $\mathrm{PR}_{\mathrm{A}}$} \\
\hline Média & 1.923 & & \\
\hline Máximo & 2.700 & Cacique Doble, Casca, Sananduva, Vanini & 1998 e $2000^{(1)}$ \\
\hline Mínimo & 1.200 & Barracão, Machadinho, Muliterno & 1997 e $1999^{(2)}$ \\
\hline \multicolumn{4}{|l|}{ Grupo $\mathrm{PR}_{\mathrm{B}}$} \\
\hline Média & 1.524 & & \\
\hline Máximo & 2.340 & Caiçara & 1998 \\
\hline Mínimo & 300 & Pinheirinho do Vale & 1999 \\
\hline \multicolumn{4}{|l|}{ Grupo $\mathrm{PR}_{\mathrm{C}}$} \\
\hline Média & 1.116 & & \\
\hline Máximo & 2.220 & Salvador das Missões & 1998 \\
\hline Mínimo & 359 & Dezesseis de Novembro & 2000 \\
\hline
\end{tabular}

(1)Cacique Doble e Sananduva, em 1998; Casca e Vanini, em 2000. (2)Muliterno, em 1997; Barracão e Machadinho, em 1999. 
tram coincidência da zona de maior risco, apontada por Cunha et al. (2001), sendo aquela onde se verificam as menores produtividades e a zona de menor risco, a de maiores produtividades. Algumas alterações neste padrão são dependentes da capacidade de água disponível dos solos (CAD) e da época de semeadura.

Ao ser realizada uma análise temporal, de 1982 a 2000, das produtividades médias dos grupos gerados com base nas produtividades médias observadas (grupos $\mathrm{PR}_{\mathrm{A}}, \mathrm{PR}_{\mathrm{B}}$ e $P R_{C}$ ), constata-se que os grupos não apresentaram alternância ao longo da série analisada (Figura 6). Ou seja, no grupo $\mathrm{PR}_{\mathrm{A}}$ foram verificados sempre as maiores produtividades ao longo de toda a série estudada, tendo os grupos $\mathrm{PR}_{\mathrm{B}}$ e $\mathrm{PR}_{\mathrm{C}}$ apresentado valores intermediários e menores, respectivamente (Figura 6). A única exceção foi o ano de 1987, em que, no grupo $\mathrm{PR}_{\mathrm{B}}$, foram verificadas produtividades médias maiores do que no grupo $\mathrm{PR}_{\mathrm{A}}$, embora a diferença entre as produtividades médias dos dois grupos tenha sido de apenas $41 \mathrm{~kg} \mathrm{ha}^{-1}$.

As produtividades da cultura da soja flutuam ao longo dos anos nos três grupos, indicando que a utilização de tecnologia na agricultura nem sempre evita perdas na produção. A ocorrência de estiagens nos anos agrícolas de 1981/1982, 1985/1986, 1988/1989, 1990/1991, 1995/1996, 1996/1997, 1998/1999 e 1999/2000, con-

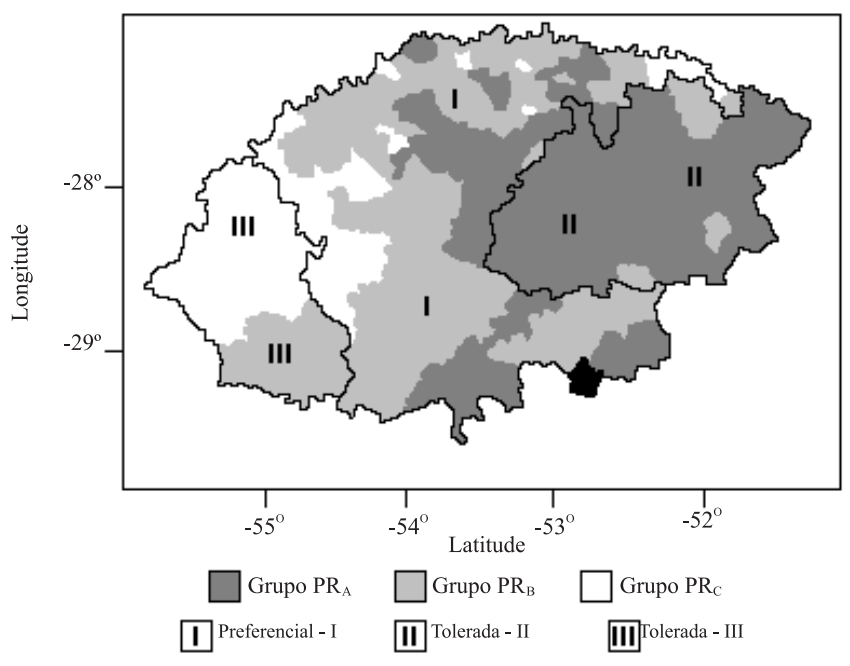

Figura 5. Municípios da região de produção significativa de soja no Rio Grande do Sul agrupados, pelo método de Ward, considerando a produtividade média, comparados ao zoneamento agroclimático para a cultura no Estado. O município em preto corresponde a Lagoão.

Pesq. agropec. bras., Brasília, v.39, n.12, p.1167-1175, dez. 2004 tribuiu para a redução das produtividades na região de estudo como um todo. Em 1990/1991, por causa da ocorrência de estiagem de abrangência regional, foi observada a maior redução nas produtividades ao longo da série estudada, atingindo igualmente os três grupos, que apresentaram valores de produtividade muito próximos. Isto demonstra que a estiagem é um fenômeno de grande escala que tem muita influência na variabilidade interanual das produtividades no Rio Grande do Sul (Bergamaschi, 1989; Berlato \& Fontana, 1999). No ano de 1999/2000, estiagem mais localizada na porção oeste da região de estudo ocasionou maiores diferenças entre as produtividades médias dos três grupos.

\section{Análise conjunta dos grupos}

Numa análise sobre os resultados dos agrupamentos das três variáveis conjuntamente, observa-se uma relação entre eles (Tabela 4). Alguns municípios que apresentam altas produtividades e alto porcentual de área cultivada de soja também estão incluídos no agrupamento de municípios de maior produção. Diversas combinações como esta podem ser feitas e a posição de um município em determinado grupo, de determinada variável, depende ou define sua colocação em outro grupo, de outra variável.

Assim, em 17,7\% dos casos houve coincidência na classificação dos municípios nos grupos, ou seja, o município foi classificado como $\mathrm{A}$, ou $\mathrm{B}$ ou ainda $\mathrm{C}$, nos três critérios de agrupamento. A maior coincidência ocorreu nos grupos A $(7,7 \%)$ e C $(7,7 \%)$, enquanto a menor ocorreu no grupo B $(2,4 \%)$.

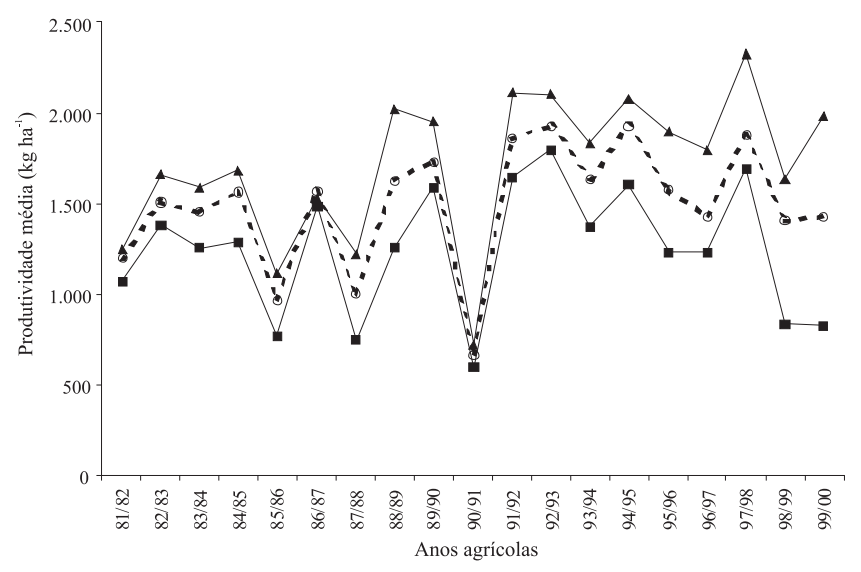

Figura 6. Produtividades médias da soja na região de produção significativa desta cultura no Rio Grande do Sul nos grupos de alto $\left(\mathrm{PR}_{\mathrm{A}}, \mathbf{\Delta}\right)$, médio $\left(\mathrm{PR}_{\mathrm{B}}, \mathrm{O}\right)$ e baixo $\left(\mathrm{PR}_{\mathrm{C}}, \boldsymbol{\square}\right)$ nível de rendimento. Período: 1982-2000. 
Tabela 4. Municípios da região de produção significativa de soja no Rio Grande do Sul agrupados segundo os critérios: produtividade (PR), produção (P) e porcentual de área cultivada com soja (A), 1997-2000 ${ }^{(1)}$.

\begin{tabular}{|c|c|c|c|c|c|c|c|}
\hline \multirow{2}{*}{ Município } & \multicolumn{3}{|c|}{ Agrupamento } & \multirow[t]{2}{*}{ Município } & \multicolumn{3}{|c|}{ Agrupamento } \\
\hline & $\overline{\mathrm{PR}}$ & $\mathrm{P}$ & $\overline{\mathrm{A}}$ & & $\overline{\mathrm{PR}}$ & $\mathrm{P}$ & $\bar{A}$ \\
\hline Água Santa & A & $\mathrm{B}$ & $\mathrm{B}$ & Cruz Alta & B & A & $\mathrm{B}$ \\
\hline Ajuricaba & $\mathrm{B}$ & $\mathrm{B}$ & $\mathrm{A}$ & David Canabarro & A & $\mathrm{C}$ & $\mathrm{C}$ \\
\hline Alecrim & $\mathrm{C}$ & $\mathrm{C}$ & $\mathrm{C}$ & Derrubadas & A & $\mathrm{B}$ & $\mathrm{C}$ \\
\hline Alegria & $\mathrm{C}$ & $\mathrm{C}$ & $\mathrm{A}$ & Dezesseis de Novembro & $\mathrm{C}$ & $\mathrm{C}$ & $\mathrm{C}$ \\
\hline Alpestre & $\mathrm{B}$ & $\mathrm{C}$ & $\mathrm{C}$ & Dois Irmãos das Missões & $\mathrm{B}$ & $\mathrm{B}$ & A \\
\hline Alto Alegre & A & $\mathrm{C}$ & $\mathrm{B}$ & Doutor Maurício Cardoso & B & $\mathrm{B}$ & A \\
\hline Ametista do Sul & $\mathrm{C}$ & $\mathrm{C}$ & $\mathrm{C}$ & Engenho Velho & A & $\mathrm{C}$ & B \\
\hline Aratiba & $\mathrm{C}$ & $\mathrm{C}$ & $\mathrm{C}$ & Entre Rios do Sul & $\mathrm{B}$ & $\mathrm{C}$ & B \\
\hline Augusto Pestana & $\mathrm{B}$ & $\mathrm{B}$ & $\mathrm{A}$ & Entre-Ijuís & $\mathrm{C}$ & A & A \\
\hline Áurea & $\mathrm{B}$ & $\mathrm{C}$ & $\mathrm{C}$ & Erebango & A & $\mathrm{B}$ & B \\
\hline Barão de Cotegipe & A & $\mathrm{C}$ & $\mathrm{C}$ & Erechim & A & $\mathrm{A}$ & $\mathrm{C}$ \\
\hline Barra do Guarita & $\mathrm{B}$ & $\mathrm{C}$ & $\mathrm{B}$ & Ernestina & A & $\mathrm{B}$ & $\mathrm{A}$ \\
\hline Barra do Rio Azul & $\mathrm{B}$ & $\mathrm{C}$ & $\mathrm{C}$ & Erval Grande & $\mathrm{B}$ & $\mathrm{C}$ & $\mathrm{C}$ \\
\hline Barra Funda & A & $\mathrm{C}$ & $\mathrm{A}$ & Erval Seco & $\mathrm{B}$ & $\mathrm{B}$ & A \\
\hline Barracão & A & $\mathrm{B}$ & $\mathrm{C}$ & Esperança do Sul & $\mathrm{B}$ & $\mathrm{C}$ & $\mathrm{B}$ \\
\hline Barros Cassal & A & $\mathrm{C}$ & $\mathrm{C}$ & Espumoso & $\mathrm{B}$ & A & B \\
\hline Benjamin Constant do Sul & B & $\mathrm{C}$ & $\mathrm{C}$ & Estação & A & $\mathrm{C}$ & B \\
\hline Boa Vista das Missões & $\mathrm{B}$ & $\mathrm{B}$ & $\mathrm{A}$ & Eugênio de Castro & $\mathrm{C}$ & $\mathrm{B}$ & B \\
\hline Boa Vista do Buricá & $\mathrm{B}$ & $\mathrm{C}$ & $\mathrm{A}$ & Faxinalzinho & A & $\mathrm{C}$ & B \\
\hline Bom Progresso & A & $\mathrm{C}$ & A & Floriano Peixoto & A & $\mathrm{C}$ & $\mathrm{C}$ \\
\hline Bossoroca & $\mathrm{C}$ & $\mathrm{B}$ & $\mathrm{C}$ & Fontoura Xavier & A & $\mathrm{C}$ & $\mathrm{C}$ \\
\hline Braga & $\mathrm{B}$ & $\mathrm{B}$ & $\mathrm{A}$ & Fortaleza dos Valos & $\mathrm{A}$ & A & B \\
\hline Cacique Doble & A & $\mathrm{C}$ & $\mathrm{C}$ & Frederico Westphalen & $\mathrm{B}$ & $\mathrm{C}$ & $\mathrm{C}$ \\
\hline Caibaté & $\mathrm{C}$ & $\mathrm{B}$ & B & Gaurama & $\mathrm{B}$ & $\mathrm{C}$ & $\mathrm{C}$ \\
\hline Caiçara & $\mathrm{B}$ & $\mathrm{C}$ & $\mathrm{C}$ & Gentil & A & $\mathrm{B}$ & $\mathrm{B}$ \\
\hline Camargo & A & $\mathrm{C}$ & $\mathrm{C}$ & Getúlio Vargas & A & $\mathrm{B}$ & B \\
\hline Campina das Missões & $\mathrm{C}$ & $\mathrm{C}$ & $\mathrm{B}$ & Giruá & $\mathrm{C}$ & A & A \\
\hline Campinas do Sul & A & A & $\mathrm{A}$ & Gramado dos Loureiros & $\mathrm{B}$ & $\mathrm{C}$ & $\mathrm{C}$ \\
\hline Campo Novo & A & $\mathrm{B}$ & $\mathrm{B}$ & Guarani das Missões & $\mathrm{C}$ & $\mathrm{B}$ & A \\
\hline Campos Borges & $\mathrm{B}$ & $\mathrm{C}$ & B & Horizontina & $\mathrm{B}$ & B & A \\
\hline Cândido Godói & $\mathrm{B}$ & $\mathrm{B}$ & $\mathrm{A}$ & Humaitá & $\mathrm{A}$ & $\mathrm{B}$ & A \\
\hline Carazinho & A & A & $\mathrm{A}$ & Ibiaçá & $\mathrm{A}$ & A & $\mathrm{B}$ \\
\hline Carlos Gomes & $\mathrm{B}$ & $\mathrm{C}$ & $\mathrm{C}$ & Ibiraiaras & $\mathrm{A}$ & $\mathrm{C}$ & $\mathrm{C}$ \\
\hline Casca & A & $\mathrm{C}$ & $\mathrm{C}$ & Ibirapuitã & $\mathrm{B}$ & $\mathrm{C}$ & $\mathrm{C}$ \\
\hline Caseiros & A & $\mathrm{C}$ & $\mathrm{C}$ & Ibirubá & A & A & $\mathrm{A}$ \\
\hline Catuípe & $\mathrm{B}$ & A & $\mathrm{A}$ & Ijuí & $\mathrm{B}$ & A & A \\
\hline Centenário & $\mathrm{B}$ & $\mathrm{C}$ & $\mathrm{C}$ & Independência & $\mathrm{B}$ & $\mathrm{B}$ & A \\
\hline Cerro Grande & $\mathrm{C}$ & $\mathrm{C}$ & $\mathrm{B}$ & Inhacorá & $\mathrm{A}$ & $\mathrm{C}$ & A \\
\hline Cerro Largo & $\mathrm{C}$ & $\mathrm{C}$ & B & Ipiranga do Sul & A & B & A \\
\hline Chapada & A & A & $\mathrm{A}$ & Irai & $\mathrm{B}$ & $\mathrm{C}$ & $\mathrm{C}$ \\
\hline Charrua & A & $\mathrm{C}$ & $\mathrm{C}$ & Itacurubi & $\mathrm{C}$ & $\mathrm{C}$ & $\mathrm{C}$ \\
\hline Chiapeta & B & A & $\mathrm{A}$ & Itatiba do Sul & $\mathrm{B}$ & $\mathrm{C}$ & $\mathrm{C}$ \\
\hline Ciríaco & B & $\mathrm{C}$ & $\mathrm{C}$ & Jaboticaba & $\mathrm{B}$ & $\mathrm{C}$ & B \\
\hline Colorado & A & $\mathrm{B}$ & $\mathrm{A}$ & Jacutinga & $\mathrm{A}$ & B & $\mathrm{A}$ \\
\hline Condor & A & A & $\mathrm{A}$ & Jarí & $\mathrm{B}$ & B & $\mathrm{C}$ \\
\hline Constantina & A & $\mathrm{B}$ & $\mathrm{B}$ & Jóia & $\mathrm{B}$ & A & B \\
\hline Coqueiros do Sul & A & B & B & Júlio de Castilhos & $\mathrm{A}$ & $\mathrm{A}$ & $\mathrm{C}$ \\
\hline Coronel Barros & $\mathrm{B}$ & $\mathrm{B}$ & $\mathrm{A}$ & Lagoa dos Três Cantos & A & B & $\mathrm{A}$ \\
\hline Coronel Bicaco & A & A & $\mathrm{A}$ & Lagoão & - & - & - \\
\hline Coxilha & A & A & $\mathrm{A}$ & Lajeado do Bugre & $\mathrm{B}$ & $\mathrm{C}$ & B \\
\hline Crissiumal & $\mathrm{B}$ & $\mathrm{B}$ & $\mathrm{A}$ & Liberato Salzano & $\mathrm{B}$ & $\mathrm{C}$ & $\mathrm{C}$ \\
\hline Cristal do Sul & $\mathrm{B}$ & $\mathrm{C}$ & $\mathrm{B}$ & Machadinho & $\mathrm{A}$ & $\mathrm{C}$ & $\mathrm{C}$ \\
\hline
\end{tabular}


Tabela 4. Continuação.

\begin{tabular}{|c|c|c|c|c|c|c|c|}
\hline \multirow[t]{2}{*}{ Município } & \multicolumn{3}{|c|}{ Agrupamento } & \multirow[t]{2}{*}{ Município } & \multicolumn{3}{|c|}{ Agrupamento } \\
\hline & PR & $\mathrm{P}$ & A & & PR & $\mathrm{P}$ & A \\
\hline Marau & A & A & $\mathrm{B}$ & Santo Antônio do Planalto & A & $\mathrm{B}$ & A \\
\hline Marcelino Ramos & $\mathrm{C}$ & $\mathrm{C}$ & $\mathrm{C}$ & Santo Augusto & A & A & A \\
\hline Mariano Moro & $\mathrm{C}$ & $\mathrm{C}$ & $\mathrm{C}$ & Santo Cristo & B & $\mathrm{B}$ & A \\
\hline Mato Castelhano & A & $\mathrm{B}$ & A & Santo Expedito do Sul & A & $\mathrm{C}$ & $\mathrm{C}$ \\
\hline Maximiliano de Almeida & $\mathrm{B}$ & $\mathrm{C}$ & $\mathrm{C}$ & São Domingos do Sul & A & $\mathrm{C}$ & $\mathrm{C}$ \\
\hline Miraguaí & $\mathrm{B}$ & $\mathrm{C}$ & $\mathrm{C}$ & São João da Urtiga & A & $\mathrm{C}$ & $\mathrm{C}$ \\
\hline Mormaço & A & $\mathrm{C}$ & $\mathrm{B}$ & São José das Missões & A & $\mathrm{C}$ & $\mathrm{B}$ \\
\hline Muliterno & A & $\mathrm{C}$ & $\mathrm{C}$ & São José do Herval & A & $\mathrm{C}$ & $\mathrm{C}$ \\
\hline Não-Me-Toque & A & A & A & São José do Inhacorá & $\mathrm{C}$ & $\mathrm{C}$ & $\mathrm{B}$ \\
\hline Nicolau Vergueiro & A & $\mathrm{B}$ & $\mathrm{B}$ & São José do Ouro & A & $\mathrm{B}$ & $\mathrm{B}$ \\
\hline Nonoai & A & $\mathrm{B}$ & $\mathrm{B}$ & São Luiz Gonzaga & $\mathrm{C}$ & A & $\mathrm{B}$ \\
\hline Nova Boa Vista & A & $\mathrm{C}$ & $\mathrm{B}$ & São Martinho & B & $\mathrm{B}$ & $\mathrm{B}$ \\
\hline Nova Candelária & $\mathrm{B}$ & $\mathrm{C}$ & $\mathrm{B}$ & São Miguel das Missões & $\mathrm{C}$ & $\mathrm{A}$ & B \\
\hline Nova Ramada & $\mathrm{B}$ & $\mathrm{B}$ & $\mathrm{A}$ & São Nicolau & $\mathrm{C}$ & $\mathrm{C}$ & $\mathrm{C}$ \\
\hline Novo Barreiro & $\mathrm{B}$ & $\mathrm{C}$ & $\mathrm{B}$ & São Paulo das Missões & $\mathrm{C}$ & $\mathrm{C}$ & $\mathrm{C}$ \\
\hline Novo Machado & $\mathrm{B}$ & $\mathrm{B}$ & $\mathrm{A}$ & São Pedro do Butiá & $\mathrm{C}$ & $\mathrm{C}$ & $\mathrm{B}$ \\
\hline Novo Tiradentes & $\mathrm{B}$ & $\mathrm{C}$ & A & São Valentim & B & $\mathrm{C}$ & $\mathrm{C}$ \\
\hline Paim Filho & A & $\mathrm{C}$ & $\mathrm{C}$ & São Valério do Sul & A & $\mathrm{C}$ & B \\
\hline Palmeira das Missões & A & A & A & Sarandi & A & $\mathrm{B}$ & $\mathrm{B}$ \\
\hline Palmitinho & $\mathrm{C}$ & $\mathrm{C}$ & $\mathrm{C}$ & Seberi & A & $\mathrm{B}$ & $\mathrm{C}$ \\
\hline Panambi & A & A & A & Sede Nova & A & $\mathrm{B}$ & A \\
\hline Passo Fundo & A & A & $\mathrm{B}$ & Selbach & A & $\mathrm{B}$ & A \\
\hline Pejuçara & A & A & A & Senador Salgado Filho & $\mathrm{C}$ & $\mathrm{C}$ & B \\
\hline Pinhal & $\mathrm{B}$ & $\mathrm{C}$ & $\mathrm{C}$ & Sertão & A & A & A \\
\hline Pinhal Grande & A & $\mathrm{B}$ & $\mathrm{C}$ & Sete de Setembro & B & $\mathrm{C}$ & A \\
\hline Pinheirinho do Vale & $\mathrm{B}$ & $\mathrm{C}$ & $\mathrm{C}$ & Severiano de Almeida & $\mathrm{C}$ & $\mathrm{C}$ & $\mathrm{C}$ \\
\hline Pirapó & $\mathrm{C}$ & $\mathrm{C}$ & $\mathrm{C}$ & Soledade & B & $\mathrm{C}$ & $\mathrm{C}$ \\
\hline Planalto & $\mathrm{B}$ & $\mathrm{C}$ & $\mathrm{C}$ & Tapejara & A & $\mathrm{B}$ & B \\
\hline Pontão & A & A & $\mathrm{A}$ & Tapera & A & $\mathrm{B}$ & A \\
\hline Ponte Preta & A & $\mathrm{C}$ & $\mathrm{C}$ & Taquaruçu do Sul & A & $\mathrm{C}$ & $\mathrm{C}$ \\
\hline Porto Lucena & $\mathrm{C}$ & $\mathrm{C}$ & $\mathrm{C}$ & Tenente Portela & B & $\mathrm{B}$ & B \\
\hline Porto Mauá & $\mathrm{B}$ & $\mathrm{C}$ & $\mathrm{B}$ & Tiradentes do Sul & $\mathrm{C}$ & $\mathrm{C}$ & B \\
\hline Porto Vera Cruz & $\mathrm{C}$ & $\mathrm{C}$ & $\mathrm{C}$ & Três Arroios & B & $\mathrm{C}$ & $\mathrm{C}$ \\
\hline Porto Xavier & $\mathrm{C}$ & $\mathrm{C}$ & $\mathrm{C}$ & Três de Maio & B & $\mathrm{A}$ & A \\
\hline Quevedos & $\mathrm{B}$ & $\mathrm{B}$ & $\mathrm{C}$ & Três Palmeiras & A & $\mathrm{B}$ & $\mathrm{B}$ \\
\hline Quinze de Novembro & A & $\mathrm{B}$ & $\mathrm{B}$ & Três Passos & B & $\mathrm{B}$ & $\mathrm{B}$ \\
\hline Redentora & $\mathrm{B}$ & $\mathrm{B}$ & $\mathrm{B}$ & Trindade do Sul & A & $\mathrm{B}$ & $\mathrm{B}$ \\
\hline Rio dos Índios & $\mathrm{B}$ & $\mathrm{B}$ & $\mathrm{B}$ & Tucunduva & B & $\mathrm{B}$ & $\mathrm{A}$ \\
\hline Rodeio Bonito & $\mathrm{B}$ & $\mathrm{C}$ & A & Tunas & B & $\mathrm{C}$ & $\mathrm{C}$ \\
\hline Ronda Alta & A & A & A & Tupanci do Sul & A & $\mathrm{C}$ & $\mathrm{B}$ \\
\hline Rondinha & A & $\mathrm{B}$ & $\mathrm{B}$ & Tupanciretã & B & A & $\mathrm{C}$ \\
\hline Roque González & $\mathrm{C}$ & $\mathrm{C}$ & $\mathrm{C}$ & Tuparendi & B & $\mathrm{B}$ & A \\
\hline Sagrada Família & A & $\mathrm{C}$ & $\mathrm{B}$ & Ubiretama & $\mathrm{C}$ & $\mathrm{C}$ & $\mathrm{B}$ \\
\hline Saldanha Marinho & A & $\mathrm{B}$ & A & Unistalda & B & $\mathrm{C}$ & $\mathrm{C}$ \\
\hline Salto do Jacuí & $\mathrm{B}$ & A & $\mathrm{B}$ & Vanini & A & $\mathrm{C}$ & $\mathrm{C}$ \\
\hline Salvador das Missões & $\mathrm{C}$ & $\mathrm{C}$ & A & Viadutos & B & $\mathrm{C}$ & $\mathrm{C}$ \\
\hline Sananduva & A & $\mathrm{B}$ & $\mathrm{C}$ & Vicente Dutra & B & $\mathrm{C}$ & $\mathrm{C}$ \\
\hline Santa Bárbara do Sul & A & A & A & Victor Graeff & A & $\mathrm{B}$ & A \\
\hline Santa Rosa & $\mathrm{B}$ & A & $\mathrm{A}$ & Vila Lângaro & A & $\mathrm{B}$ & $\mathrm{B}$ \\
\hline Santiago & $\mathrm{B}$ & $\mathrm{B}$ & $\mathrm{C}$ & Vila Maria & A & $\mathrm{C}$ & $\mathrm{C}$ \\
\hline Santo Ângelo & $\mathrm{B}$ & A & A & Vista Alegre & B & $\mathrm{C}$ & $\mathrm{C}$ \\
\hline Santo Antônio das Missões & $\mathrm{C}$ & $\mathrm{B}$ & $\mathrm{C}$ & Vista Gaúcha & B & $\mathrm{C}$ & A \\
\hline Santo Antônio do Palma & A & $\mathrm{C}$ & $\mathrm{C}$ & Vitória das Missões & $\mathrm{C}$ & $\mathrm{C}$ & $\mathrm{B}$ \\
\hline
\end{tabular}

${ }^{(1)}$ Os índices A, B e C representam, para cada critério de agrupamento, o grupo alto, médio e baixo, respectivamente, em que o município situou-se. 


\section{Conclusões}

1. A análise de agrupamento pelo método de Ward é um bom instrumento para definir regiões homogêneas de produção, rendimento e porcentual de área cultivada com soja no Estado do Rio Grande do Sul.

2. A definição de grupos homogêneos permite um conhecimento mais detalhado da produção de soja no Estado do Rio Grande do Sul.

\section{Agradecimentos}

Ao Conselho Nacional de Desenvolvimento Científico e Tecnológico (CNPq), pelo apoio financeiro.

\section{Referências}

ÁVILA, A.M.H.; BERLATO, M.A.; SILVA, J.B.; FONTANA, D.C. Probabilidade de ocorrência de precipitação pluvial mensal igual ou maior que a evapotranspiração potencial para a estação de crescimento das culturas de primavera-verão no Estado do Rio Grande do Sul. Pesquisa Agropecuária Gaúcha, Porto Alegre, v.2, p.149-154, 1996.

BERGAMASCHI, H. Variations on the Brazilian soybean production related to the drought occurrences: preliminary analysis. In. WORLD SOYBEAN RESEARCH CONFERENCE, 4. 1989. Buenos Aires. Anais. Buenos Aires: Asociación Argentina de la Soja, 1989. v.5, p.2153-2158.

BERLATO, M.A.; FONTANA, D.C. Variabilidade interanual de precipitação pluvial e rendimento de soja no Estado do Rio Grande do Sul. Revista Brasileira de Agrometeorologia, v.7, p.119-125, 1999.

BERLATO, M.A.; FONTANA D.C. EI Niño e La Niña: Impactos no clima, na vegetação e na agricultura do Rio Grande do Sul. Aplicações de previsões climáticas na agricultura. Porto Alegre: Ed. da UFRGS, 2003110 p.

CUNHA, G.R.; ASSAD, E.D. Uma visão geral do número especial da RBA sobre o zoneamento agrícola no Brasil. Revista Brasileira de Agrometeorologia, v.9, p.446-459, 2001. Número Especial: Zoneamento Agrícola.

CUNHA, G.R.; HASS, J.C. Zoneamento agrícola: recomendações de épocas de semeadura de soja para o estado do Rio Grande do Sul - safra 1996/97. Passo Fundo: Embrapa-CNPT, 996. 28p. (Documentos, 29).

CUNHA, G.R.; BARNI, N.A.; HASS, J.C.; MALUF, J.R.T; MATZENAUER, R.; PASINATO, A.; PIMENTEL, M.B.M.; PIRES, J.L.F. Zoneamento agrícola e época de semeadura para soja no Rio Grande do Sul. Revista Brasileira de Agrometeorologia, v.9, p.446-459, 2001. Número Especial: Zoneamento Agrícola.

EMATER/RS. Informações institucionais, técnicas, notícias, projetos, publicações e serviços. Disponível em <http://www.emater.tche.br> Acesso em: 01 fev. 2002.

FEDERAÇÃO DAS ASSOCIAÇÕES DE MUNICÍPIOS DO RIO GRANDE DO SUL. Informações institucionais, produtos, notícias, pesquisas, eventos e serviços. Disponível em: <http://dados.famurs.com.br/portal> Acesso em: 24 ago. 2001.

IBGE. Informações institucionais, produtos, notícias, pesquisas, eventos e serviços. Disponível em: 〈http://www.sidra.ibge.gov.br/bda〉 Acesso em: 30 ago. 2001.

MATZENAUER, R.; BERGAMASCHI, H.; BERLATO, M.A.; MALUF, J.R.T.; BARNI, N.A.; BUENO, A.C.; DIDONE, I.A.; ANJOS, C.S.; MACHADO, F.A.; SAMPAIO, M.R. Consumo de água e disponibilidade hídrica para milho e soja no Rio Grande do Sul. Porto Alegre: Fepagro, 2002. 105p. (Boletim FEPAGRO, 10).

MOTA, F.S.; BEIRSDORF, M.I.C.; ACOSTA, M.J.C.; MOTTA, W.A.; WESTPHALEN, S.L. Zoneamento agroclimático do Rio Grande do Sul e Santa Catarina. Pelotas: Ipeas, 1974. 122p. (Circular, 50).

RIO GRANDE DO SUL. Secretaria da Agricultura e Abastecimento. Centro Nacional da Pesquisa do Trigo. Macrozoneamento agroecológico e econômico do Estado do Rio Grande do Sul. Porto Alegre, 1994. v.2.

WILKS, D.S. Statistical methods in the atmospheric sciences: an introduction. New York: Academic Press, 1995. 467p.

Recebido em 4 de junho de 2004 e aprovado em 17 de agosto de 2004 